\title{
Cryo-electron microscopy reconstructions of two types of wild rabbit hemorrhagic disease viruses characterized the structural features of Lagovirus
}

\author{
Zhongjun $\mathrm{Hu}^{1 *}$, Xiaojuan Tian ${ }^{1,2^{*}}$, Yujia Zhai ${ }^{1}$, Wei Xu${ }^{1}$, Dong Zheng ${ }^{2}(\bowtie)$, Fei Sun ${ }^{1}(\bowtie)$ \\ 1 National Laboratory of Biomacromolecules, Institute of Biophysics, Chinese Academy of Sciences, \\ Beijing 100101, China \\ 2 Department of Biochemistry and Molecular Biology, College of Life Sciences, Beijing Normal University, \\ Beijing 100875, China \\ $\triangle$ Correspondence: feisun@ibp.ac.cn (F. Sun), zhengd@bnu.edu.cn (D. Zheng) \\ Received October 9, 2009; accepted November 7, 2009
}

\section{ABSTRACT}

Rabbit hemorrhagic disease was described in China in 1984 and can cause hemorrhagic necrosis of the liver within two or three days after infection. The etiological agent, rabbit hemorrhagic disease virus (RHDV), belongs to the Lagovirus genus in the Caliciviridae family. Compared to other calicivirus, such as rNV and SMSV, the structure of Lagovirus members is not well characterized. In this report, structures of two types of wild RHDV particles, the intact virion and the core-like particle (CLP), were reconstructed by cryo-electron microscopy at $11 \AA$ and $17 \AA$, respectively. This is the first time the 3D structure of wild caliciviruses CLP has been provided, and the 3D structure of intact RHDV virion is the highest resolution structure in Lagovirus. Comparison of the intact virion and CLP structures clearly indicated that CLP was produced from the intact virion with the protrusion dissociated. In contrast with the crystal structures of recombinant Norovirus and San Miguel sea lion virus, the capsomers of RHDV virion exhibited unique structural features and assembly modes. Both P1 and $P 2$ subdomains have interactions inside the $A B$ capsomer, while only $\mathrm{P} 2$ subdomains have interaction inside CC capsomer. The pseudo atomic models of RHDV capsomers were constructed by homology modeling and density map fitting, and the rotation of RHDV VP60 P domain with respect to its $S$ domain, compared with
SMSV, was observed. Collectively, our cryo-electron microscopic studies of RHDV provide close insight into the structure of Lagovirus, which is important for functional analysis and better vaccine development in the future.

KEYWORDS rabbit hemorrhagic disease virus, calicivirus, Lagovirus, core-like particle, cryo-electron microscopy reconstruction

\section{INTRODUCTION}

Rabbit hemorrhagic disease (RHD), a viral disease characterized by high mortality in adult rabbits, was first described in China (Liu et al., 1984) and had spread all over the world within a few years (Nowotny et al., 1997). The disease was highly infectious for domestic and wild rabbits, and had great impact on relevant economy and ecology. The typical pathological symptom is hemorrhagic necrosis of the liver within two or three days after infection (Xu and Chen, 1989).

Its etiological agent, the rabbit hemorrhagic disease virus (RHDV), is a member of the Lagovirus genus in the Caliciviridae family (Ohlinger and Thiel, 1991). RHDV is a positive-sense, single-stranded RNA virus (Meyers et al., $1991 \mathrm{~b})$. Its genomic RNA is $\sim 7.5 \mathrm{~kb}$ in length and contains two open reading frames (ORF1 and ORF2) (Martin Alonso et al., 1996; Wirblich et al., 1996). The ORF1 encodes the primary polypeptide with molecular weight $\sim 257 \mathrm{kDa}$, which is cleaved

"These authors contributed equally to this work. 
into the non-structural viral proteins and the major capsid protein VP60 at the $\mathrm{C}$-terminus. The ORF2 encodes the minor capsid protein VP10. After infecting cells, a 2.2-kb subgenomic RNA encoding VP60 and VP10 is transcribed. Either genomic or sub-genomic RNA can be covalently linked to a small protein VPg $(10-15 \mathrm{kDa})$ at the 5'-end (Meyers et al., 1991a) and be packaged into the virion.

The RHDV virion is a non-enveloped particle with a diameter of $32-40 \mathrm{~nm}$. It exhibits characteristic cup-shaped depressions on the surface with $T=3$ icosahedral symmetry, which was revealed by negative staining electron microscopy (Valicek et al., 1990). As calicivirus, the shell of RHDV virion is mainly composed of major capsid protein VP60 (Clarke and Lambden, 1997). Recombinant VP60 produced by baculovirus expression system is able to self-assemble into the virus-like particles (VLPs) that were indistinguishable in morphology from authentic infectious virion (Laurent et al., 1994).

In some cases when the subacute/chronic RHD evolves 4-8 days post-infection and is followed by death, or more often, by recovery, RHDV core-like particles (CLP) were found in liver and spleen but not in bloodstream (Barbieri I et al., 1997; Antonio and Lorenzo, 2008). This particle had a smooth surface with $25-29 \mathrm{~nm}$ diameter (Alexandrov et al., 1993; Granzow et al., 1996). The molecular weight of CLP subunit was about $28-30 \mathrm{kDa}$ corresponding to the $\mathrm{N}$-terminal part of VP60. The CLP had no haemagglutinating property but could react with RHDV convalescent rabbit sera and monoclonal antibodies of VP60 N-terminus (Barbieri I et al., 1997; Antonio and Lorenzo, 2008). It was proposed that the genesis of CLP was due to a degradative process, which was possibly the consequence of physiological clearance of the RHDV-IgM immuno-complex that formed in large amounts at the beginning of the humoral response (Barbieri I et al., 1997; Antonio and Lorenzo, 2008). In addition to RHDV, similar CLP was also discovered in wild amyelois chronic stunt virus (ACSV) (Hillman et al., 1982). Furthermore, the recombinant $\mathrm{N}$-terminal region (1-227) of Norovirus (NV) capsid protein was found to assemble into CLP-like particles (BertolottiCiarlet et al., 2002).

During the past decades, several calicivirus structures have been reported. Examples include the cryo-electron microscopy (cryo-EM) reconstruction of the primate calicivirus $(\sim 25 \AA)$ (Prasad et al., 1994), San Miguel sea lion virus (SMSV) (22 A) (Chen et al., 2004) and RHDV (32 A) (Zheng et al., 2001). In addition, the recombinant VP60s of NV (Venkataram Prasad et al., 2000; Bertolotti-Ciarlet et al., 2002), Grimsby virus (GrV) (Chen et al., 2004), Parksville virus (PV) (Chen et al., 2004) and RHDV (Barcena et al., 2004) were heterogeneously expressed and assembled into VLPs whose 3D structures were successfully reconstructed by cryo-EM with the resolution about $20 \AA$. In addition, higher resolution cryo-EM reconstructions of Feline calicivirus (FCV) VLP (16 Å) (Bhella et al., 2008) and Murine Norovirus (MNV)
VLP (12 $\AA$ ) (Katpally et al., 2008), as well as the interactions between virion and receptor or antibody, fJAM-1-labeled FCV (18 $\AA$ ) (Bhella et al., 2008), MNV-Fab (22 A) (Katpally et al., 2008) and RHDV VLP/mAb-E3 (32 $\AA$ ) (Thouvenin et al., 1997), were reported recently. There are only two available atomic crystal structures of the calicivirus, the recombinant NV VLP (Prasad et al., 1999) and the wild SMSV (Chen et al., 2006). These structures exhibit $T=3$ icosahedral symmetry and comprise 180 capsid proteins that are organized into 90 dimeric capsomers. The structures revealed that the major capsid protein has two principal domains: a shell (S) domain with a typical eight-stranded $\beta$-barrel fold and a protrusion $(P)$ domain that can be further divided into $\mathrm{P} 1$ and $\mathrm{P} 2$ subdomains. The $S$ domain is well conserved among the caliciviruses, while the $\mathrm{P} 1$ sub-domain is only moderately conserved and the $\mathrm{P} 2$ sub-domain is highly variable.

This knowledge revealed the common structural assembly feature of caliciviruses. The icosahedral asymmetrical unit of calicivirus capsid is composed of three quasi-equivalent subunits designated $A, B$ and $C$ (Rossmann and Johnson, 1989) according to the position in $T=3$ icosahedron. The $A / B$ dimers are located at the local 2-fold axes, while the $\mathrm{C} / \mathrm{C}$ dimers are located at the icosahedral 2-fold axes. Three A/B and three $\mathrm{C} / \mathrm{C}$ dimers alternately arrange around icosahedral 3 -fold axes, while five A/B dimers arrange around icosahedral 5 -fold axes.

There are four subfamilies in the Caliciviridae family, Lagovirus, Norovirus, Vesivirus and Sapovirus. Despite of the structural knowledge of the other three subfamilies, the structure of Lagovirus is not well characterized. In this report, structures of two types of wild RHDV particles, intact virion and the core-like particle (CLP), were reconstructed to $11 \AA$ and $17 \AA$, respectively. This is the first $3 D$ structural characterization of wild caliciviruses CLP and the highest resolution 3D structure of intact RHDV virion in Lagovirus. Structural comparison between intact virion and CLP and comparison between RHDV and other calicivirus (NV and SMSV) made the structural features of Lagovirus much clearer than before.

\section{RESULTS AND DISCUSSION}

\section{Purified wild RHDV particles contain VP60, VP10 and VPg}

The SDS-PAGE of the purified RHDV particles shows that the wild virion contains major capsid proteins VP60, minor capsid proteins VP10 and genome-linked proteins VPg (Fig. 1A). There are also several bands around $45 \mathrm{kDa}$ that were viral species as discussed before (Granzow et al., 1996) instead of contaminants. Before cryo-EM analysis, the purified RHDV samples were examined by negative staining electron microscopy. These RHDV particles showed almost identical non-membrane-embedded morphology with icosahedral symmetry and same size of $\sim 40 \mathrm{~nm}$ diameter (Fig. 1B). The 

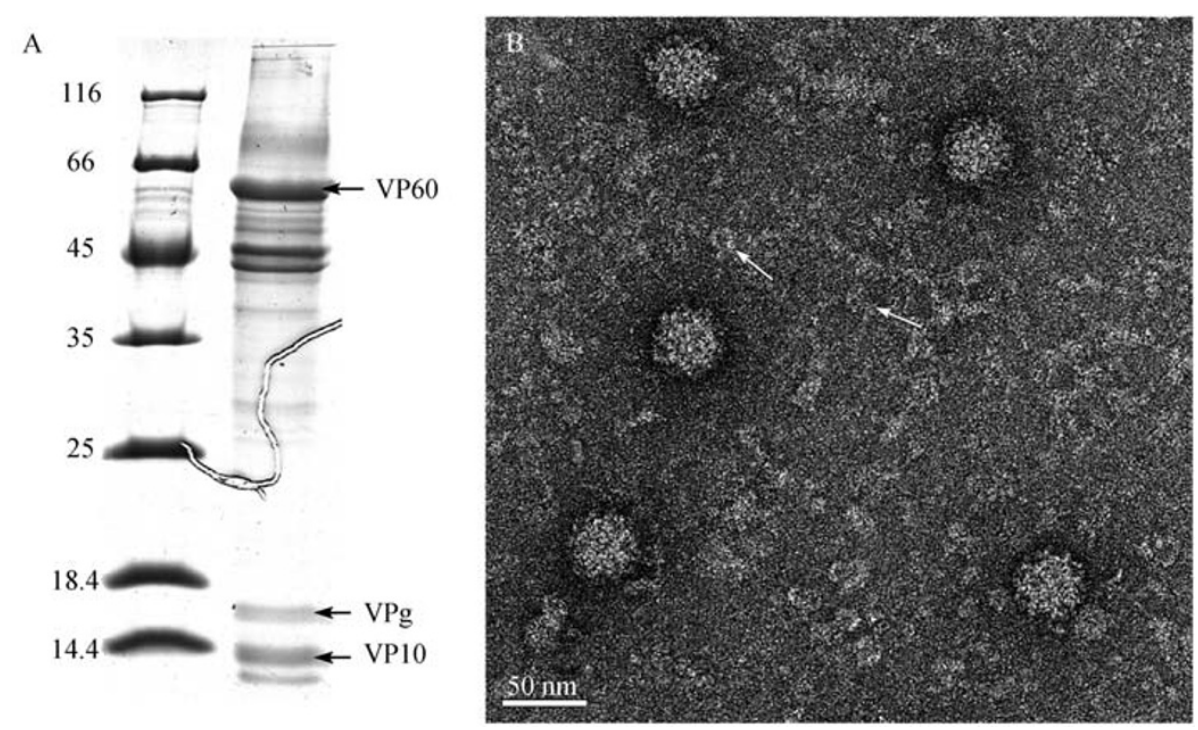

Figure 1. Analysis of purified RHDV particles by SDS-page and negative staining electron microscopy. (A) SDS-PAGE of the purified RHDV. Left lane: protein molecular weight marker. VP60, VP10 and VPg are labeled by arrows. (B) Negative staining electron micrograph of RHDV particles (Scale bar $=50 \mathrm{~nm}$ ). The small particles indicated by white arrow might be the ferritin-like structures particles.

obvious cup-shaped depressions were observed, confirming that the wild RHDV virion structures are complete. There were also some small particles with the diameter $\sim 10 \mathrm{~nm}$ (Fig. 1B) by negative staining. They might be the ferritin-like structures as previously reported (Valicek et al., 1990).

\section{Four types of RHDV particles were detected by cryo- electron microscopy}

To further investigate the native structure, the purified RHDV particles were embedded in thin vitreous ice by fast freezing and imaged by cryo-EM in low-dose mode. Four types of particles can be observed in the raw cryo-EM micrograph according to the diameters of the whole virions and the density differences inside the capsid shell (Fig. 2A). The intact virion containing whole genomic RNA showed high density inside (HV), while the one containing sub-genomic RNA showed low density inside (LV). The small particle with inner high density $(\mathrm{HC})$ was believed to be the core-like particle (CLP) with whole genomic RNA, while the small particle with inner low density (LC) was thought to be the one with subgenomic RNA. From $\sim 230$ cryo-electron RHDV micrographs recorded, 16,785 particles were picked out and $\sim 5 \%$ CLPs ( $\mathrm{HC}$ or LC) were identified, as previously reported (Antonio and Lorenzo, 2008). The presence of two types of intact virions with high or low density inside was consistent with our previous experiments (Zheng et al., 2001). The existence of CLP in purified RHDV was also reported by negative staining (Alexandrov et al., 1993), but this is the first time to observe two types of CLPs with high or low density inside.

\section{Three-dimensional reconstruction of intact and core-like virions}

Based on the 2D image analysis above, 15,890 intact RHDV particles (HV and LV) and $895 \mathrm{CLPs}(\mathrm{HC}$ and LC) were picked out individually and processed for $3 \mathrm{D}$ reconstructions. The effective resolutions for RHDV map (Fig. 2B) and CLP map (Fig. 2C) are about $11 \AA$ and $17 \AA$, respectively, based on the 0.5 cutoff of Fourier shell correlation coefficient (FSC) among independent reconstructions. Because the RNA density inside each particle was considered as noise for icosahedral reconstruction, HV and LV or HC and LC particles were not distinguished during image processing.

The diameter of intact RHDV virion is $\sim 41 \mathrm{~nm}$ according to the reconstructed map (Fig. 2B). The intact virion is composed of 180 capsid proteins VP60 that are organized into 90 arch-like dimeric capsomers. Those capsomers are arranged on a $\mathrm{T}=3$ icosahedral lattice and form a contiguous shell with 32 cupshaped surface depressions. In accord with other $\mathrm{T}=3$ caliciviruses, the icosahedral asymmetrical unit of RHDV capsid consists of three quasi-equivalent subunits $A, B$ and $C$ (Rossmann and Johnson, 1989). The $A$ and $B$ subunits assemble into the $A / B$ dimeric capsomer ( $A B$ capsomer). and two $C$ subunits assemble into the $C / C$ dimeric capsomer (CC capsomer). Thirty $\mathrm{CC}$ capsomers are located at the icosahedral 2 -fold axes while the rest of $60 \mathrm{AB}$ capsomers are located at the local pseudo 2-fold axes. The surface depressions formed by 90 capsomers are located at the icosahedral 5 - and 3-fold axes. Every three $A B$ capsomers and three $C C$ capsomers alternately arrange around the icosahedral 3 -fold axes, yielding a 

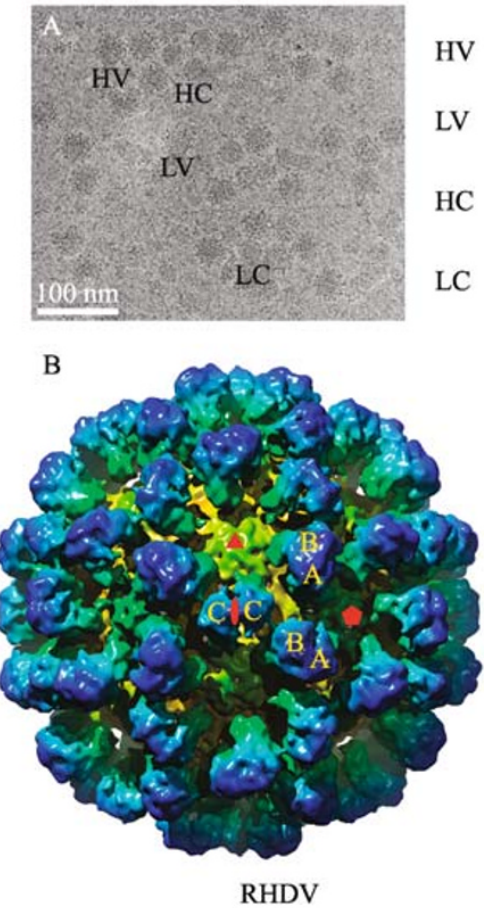

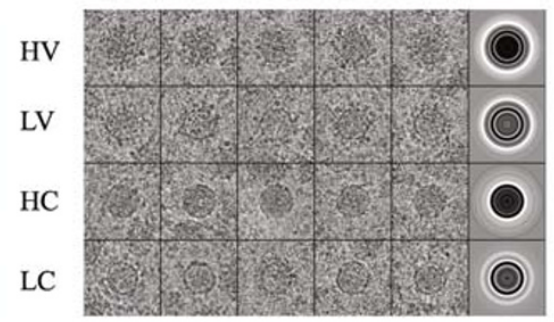

C

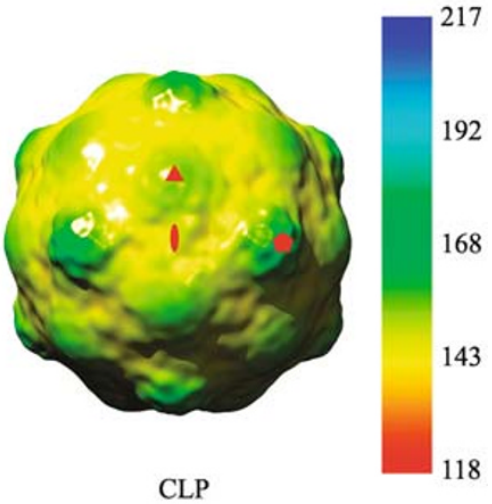

Figure 2. Cryo-electron microscopy of the intact RHDV virion and CLP. (A) The raw cryo-EM micrograph (Left) contains four types of particles: intact virions with high density (HV) or low density inside (LV) and CLPs with high density (HC) or low density inside (LC) $($ scale bar $=100 \mathrm{~nm}$ ). Those four types of particles were picked out separately (Right, the left five columns) and rotationally averaged (Right, the right column). (B) and (C) The reconstructed maps of intact RHDV virion and CLP with diameter $\sim 41 \mathrm{~nm}$ and $\sim 32 \mathrm{~nm}$, respectively, are represented as isosurface. The icosahedral 5-, 3- and 2-fold axes are indicated by red symbols. AB and CC capsomers are labeled by yellow. The color bar represents the different radii with four color gradients from red (118 $\AA$ ) to yellow $(143 \AA)$, to green $(168 \AA)$, to cyan $(192 \AA)$ and to blue $(217 \AA)$.

local pseudo 6-fold symmetry, while every five AB capsomers arrange around the icosahedral 5 -fold axes. The high-resolution cryo-EM map of wild RHDV clearly demonstrates that the depressions located at the 3-fold axes are much deeper than the one located at the 5 -fold axes.

The diameter of CLP is $\sim 32 \mathrm{~nm}$ according to the reconstructed map (Fig. 2C). Like the wild RHDV virion, CLPs also exhibit $T=3$ icosahedral symmetry. The surface of CLPs is largely smooth but has the protruding structural features at the 5-fold and 3 -fold axes, and the 5-fold protrusions are much bigger than the 3 -fold ones. At the same time, on the 2-fold axes, no arch-like capsomer structures were observed on the surface of CLPs.

In summary, from the purified RHDV samples, two types of 3D structures were constructed, and they exhibited completely different features. The structures of RHDV intact virion and CLP were further compared and analyzed next to find their biological relevance.

\section{Comparison between intact RHDV virion and CLP}

From the central section vertical to one of 2-fold axes (Fig 3A, left), it is clear that the intact RHDV virion contains four parts of densities. From center to outside, they are RNA containing core (O region), inner shell (IS region), capsid shell (S region) and capsid protrusion (P region), respectively. In contrast, the CLP only has three parts of densities without the capsid protrusion density. More accurate density comparison between intact virion and CLP was carried by calculating their azimuthally averaged density distributions as a function of radius (the radial density profiles). When such profiles of the intact virion and CLP were plotted in the same diagram and compared (Fig $3 \mathrm{~A}$, right), both the radical density distributions upon the $O$ region ( $0-90 \AA$ radius), IS region $(90-120 \AA$ radius $)$ and $S$ region (120-150 Å radius) are nearly identical. However, the density distribution (150-220 radius) corresponding to the $P$ region in the intact virion was not detectable on the profile of CLP. The similar density distribution profile from the $O$ region to the $S$ region reveals that the small virion CLP may be produced from the intact virion when protruding capsomers are dissociated. The high densities in the $O$ regions of both types of particles are due to the contained genomic or sub-genomic RNA as previously reported (Zheng et al., 2001).

The nanometer resolution reconstruction of intact RHDV virion $(11 \AA)$ enabled us to fit the RHDV VP60 atomic 

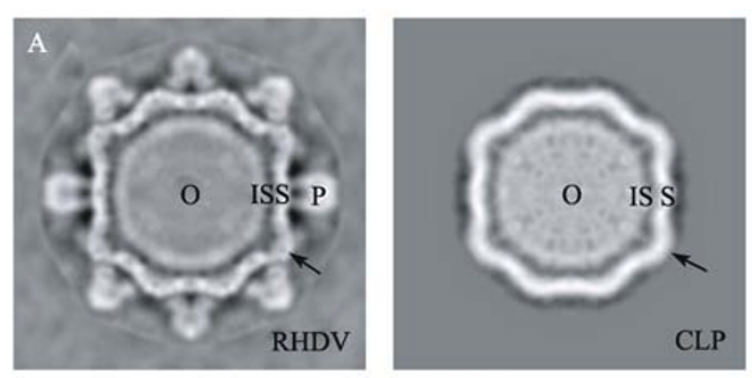

B

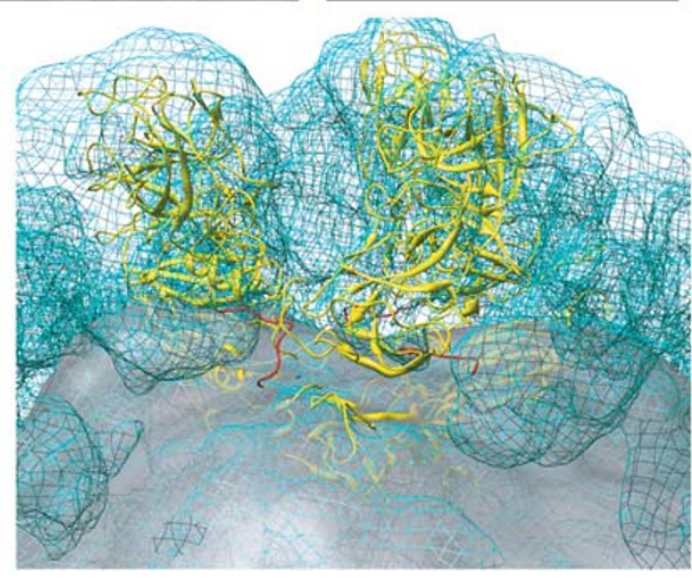

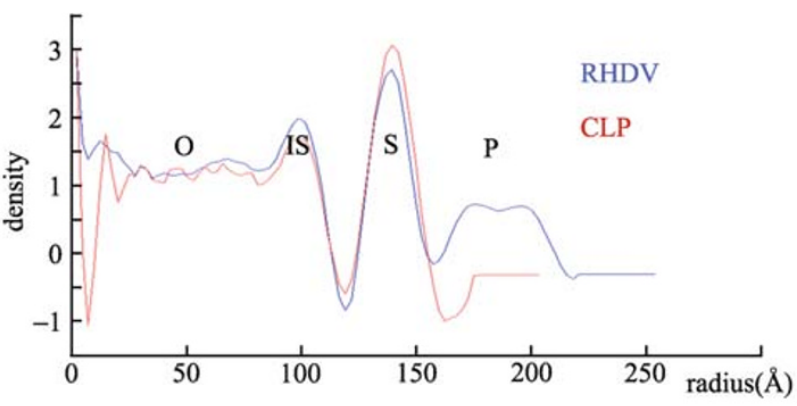

$\mathrm{C}$

\begin{tabular}{|c|c|c|}
\hline & 220 & 230 \\
\hline RHD & \multirow{7}{*}{\multicolumn{2}{|c|}{ 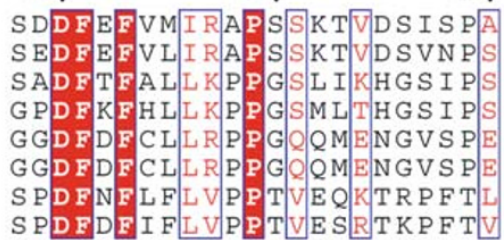 }} \\
\hline & & \\
\hline & & \\
\hline FCV-F9 & & \\
\hline Sapporo & & \\
\hline & & \\
\hline GrV & & \\
\hline
\end{tabular}

Figure 3. Comparison between intact RHDV virion and CLP. (A) Left panel, the central section's perpendicular to icosahedra 2fold axe were plotted against the intact RHDV virion and CLP with each region $(\mathrm{O}, \mathrm{IS}, \mathrm{S}$ and $\mathrm{P})$ labeled and the arrows indicate the similar features between two structures. Right panel, the azimuthally averaged density distributions ( $y$-axis) of intact RHDV virion and CLP are plotted as the function of the radius (x-axis). (B) The RHDV VP60 atomic coordinates modeled by SMSV crystal structure are fitted into RHDV cryo-EM maps. The intact RHDV virion is displayed by cyan mesh and CLP map by gray semitransparent surface. The $S$ and $P$ domain of RHDV are colored by yellow and the loop between $S$ and $P$ domains by red. (C) Multiple sequence alignment upon the loop domains of caliciviruses major capsid protein VP60 (see Materials and Methods). For clarity, only 8 sequences, including RHDV NJ85 and European brown hare syndrome virus (EBHSV) from Lagovirus, SMSV4 and FVC-F9 from Vesivirus, Sapporo virus and PV from Sapovirus and NV and GrV from Norovirus, are shown.

coordinates that were modeled by SMSV crystal structure (pdb code 2GH8) into the cryo-EM map (Fig. 3B). In this fine fitness, the dimeric capsomer could be separated into two halves under such resolution, and each half could be fitted well by the P domain of VP60. The S domain of VP60 could be fitted into the $S$ region while the low-density gap between $S$ region and $P$ region is just corresponding to the loop (220-240) called jungle domain of VP60. Combined with the above radial density profiles, it is obvious that the capsid shell of intact virion is composed of S domains of VP60, and the protruding capsomers are composed of $\mathrm{P}$ domains of VP60, while the capsid shell of CLP is only composed of 180 VP60 S domains. The $\mathrm{P}$ domain dissociating place from intact virion to CLP is located at the jungle domain of VP60. Alignment of multiple sequences of caliciviruses major capsid proteins VP60 (Fig. 3C) indicated that the jungle domain of RHDV VP60 is conserved among the caliciviruses. Therefore, the dissociating phenomena of VP60 P domain may be a common physiological mechanism for caliciviruses.

All VP60 mutants with deletion at C-terminal region that is located in P domain of VP60 are unable to assemble into VLP (Barcena et al., 2004), suggesting that the small virion CLP could not be directly assembled by $S$ domains of VP60. One reasonable explanation for CLP occurrence is that the protruding capsomers are dissociated from the intact RHDV virion to form CLP. It is reported that such degradation of intact virion and CLP formation was possibly due to the physiological clearance of the RHDV-IgM immuno-complex (Barbieri et al., 1997; Antonio and Lorenzo, 2008). In accordance, this point is also proved by the fact that the intact ACSV particles could be changed to ACSV CLP after incubation with $\alpha$ chymotrypin (Hillman et al., 1982). The SDS-PAGE of purified RHDV sample (Fig. 1A) showed that the relatively weak band $(\sim 30 \mathrm{kDa})$ would refer to the $S$ domains of RHDV major capsid proteins that assemble into RHDV CLP.

Although it was reported that the recombinant NV S domain ( $\mathrm{N}$ terminal 1-227) is able to assemble into CLP-like particle, its diameter $(\sim 27 \mathrm{~nm})$ is smaller than that of expected icosahedral shell $(30 \mathrm{~nm})$ of the rNV VLP particles (Bertolotti-Ciarlet et al., 2002). In contrast, RHDV CLP could not be 
directly assembled from the $S$ domain and its diameter is exactly identical to that of the icosahedral shell of intact RHDV particle. This comparison suggests that the recombinant NV CLP like particle does not represent the wild NV CLP and the $3 D$ reconstruction of RHDV CLP reported here is the first 3D structure of wild caliciviruses CLP.

\section{Comparison of capsomers among RHDV, rNV and SMSV indicated the capsid proteins assembly differences among caliciviruses}

The calicivirus family contains four sub-families: Lagovirus, Vesivirus, Sapovirus and Norovirus. In addition to the previous low-resolution cryo-EM maps, there are only two high-resolution crystal structures available in this family. They are the crystal structure of $r N V$ (pdb code: $1 \mathrm{IHM}$ ), a member of Norovirus sub-family, and the crystal structure of SMSV (pdb code: 2GH8), a member of Vesivirus sub-family. Here, we reconstructed the intact RHDV from Lagovirus to $11 \AA$, which is the highest resolution among the current cryo-EM reconstructions of caliciviruses. Comparison of these three available high-resolution calicivirus structures shows that capsid proteins assemble differently among Lagovirus, Norovirus and Vesivirus.

The 3.4- $\AA$ crystal structure of rNV (Prasad et al., 1999) and 3.2-Å crystal structure of SMSV (Chen et al., 2006) were used to calculate the corresponding density maps by the program "pdb2mrc" from EMAN suite (Ludtke et al., 1999). Both maps are low-pass filtered to the resolution of $11 \AA$ for consensus comparison with cryo-EM map of RHDV (Fig. 4A). It is known that the major capsid protein VP60 of caliciviruses is composed of two principal domains, a shell (S) domain and a protrusion $(P)$ domain. The $S$ domain is involved in the formation of the contiguous icosahedral shell, and the $P$ domain is further divided into two subdomains, P1 and P2. P1 subdomain forms the leg of the protrusion and $P 2$ forms the top. P2 subdomain shows obvious structural differences among RHDV, rNV and SMSV. RHDV has a hat-shaped prominency at $\mathrm{P} 2$ region, while $\mathrm{rNV}$ has an approximately rectangular platform on its top and SMSV exhibits an approximately parallelogram-shaped platform with a dent at the center as previously reported (Chen et al., 2004). The different shapes of P2 region implicate the difference in antigen structures and antibody binding sites of caliciviruses.

The interaction among adjacent capsomers also exhibits differences (Fig. 4A and 4B). For SMSV, CC capsomer interacts with two adjacent $A B$ capsomers via $P 1$ subdomains. However, for RHDV and rNV, such interactions are mediated via P2 subdomains. Interestingly, CC capsomer of $\mathrm{rNV}$ interacts with all the four adjacent $A B$ capsomers while CC capsomer of RHDV or SMSV interacts with only two adjacent $A B$ capsomers and no interactions were observed with two other adjacent $A B$ capsomers. On the other hand, the arrangements for $\mathrm{CC}$ capsomer and two interacting adjacent
$A B$ capsomers have different orientations between RHDV and SMSV. The interactions of two VP60 proteins within the dimeric capsomer were also compared among RHDV, rNV and SMSV (Fig. 4B). In both CC capsomer and AB capsomer of $r N V$, the two VP60 proteins interact with each other via wide contacts from P2 subdomain to P1 subdomain. For SMSV, only P2 subdomains mediate such interaction. However, for RHDV, the dimeric interactions show more difference between $C C$ capsomer and $A B$ capsomer. In CC capsomer, only $\mathrm{P} 2$ subdomains are involved in dimeric interaction like SMSV, but in AB capsomers, both P1 and P2 subdomains mediate the interaction, and there is an obvious hole between $\mathrm{P} 1$ and $\mathrm{P} 2$ region that was not detectable in rNV capsomers.

The specific packing among 90 RHDV capsomers and different dimeric interactions within capsomers implies that the $\mathrm{P}$ domain of RHDV VP60, especially the P2 subdomain, has different fold and conformation in comparison with other caliciviruses. Such differences were further investigated by EM map-based model building (Fig. 5). The quasi-atomic coordinates of RHDV major capsid protein VP60 were modeled according to crystal structure of SMSV VP60 (Chen et al., 2006) and split into S domain, P1 subdomain and P2 subdomain for cryo-EM map fitting. The fitness between $\mathrm{P}$ domain and $\mathrm{CC}$ capsomer protrusion density, between $S$ domain and $C C$ capsomer $S$ region density, between $\mathrm{P} 1$ subdomain and $\mathrm{AB}$ capsomer $\mathrm{P} 1$ region density, and between $S$ domain and $A B$ capsomer $S$ region density shows high accuracy under such resolution, and their fittings could be further optimized by automatic local minimization algorithm. However, the fitting between P2 subdomain and $A B$ capsomer $P 2$ region density could not be achieved by auto fitting method and requires manual approach. The fitness was in relatively lower quality, indicating that the RHDV VP60 P2 subdomain has big differences with SMSV.

After fitting two $\mathrm{P}$ domains and two $\mathrm{S}$ domains into $\mathrm{CC}$ capsomer density or fitting two P1 subdomains, two P2 subdomains and two $S$ domains into $A B$ capsomer density, we obtained the quasi-atomic model of the RHDV CC capsomer or AB capsomer with dimeric major capsid proteins (Fig. 5). In accordance with the analysis of capsomer packing from the RHDV intact virion described above, the modeling fitting further demonstrates that two monomers in the CC capsomer are precisely correlated by the icosahedral 2-fold axis, while two monomers in $A B$ capsomer are linked approximately by the local pseudo 2-fold axis. Further comparison of the monomer in $A B$ capsomer with the monomer in $\mathrm{CC}$ capsomer shows different orientations of $\mathrm{P} 1$ and $\mathrm{P} 2$ subdomains using $\mathrm{S}$ domain as reference, implying the big conformational change between $A B$ capsomer and CC capsomer. In addition, the SMSV VP60 structure was superposed to $A B$ capsomer or $C C$ capsomer monomer by matching their $S$ domains (the most conserved domain). Furthermore, we found that there was a rotation of the RHDV VP60 P domain with respect to its $S$ domain, 
A
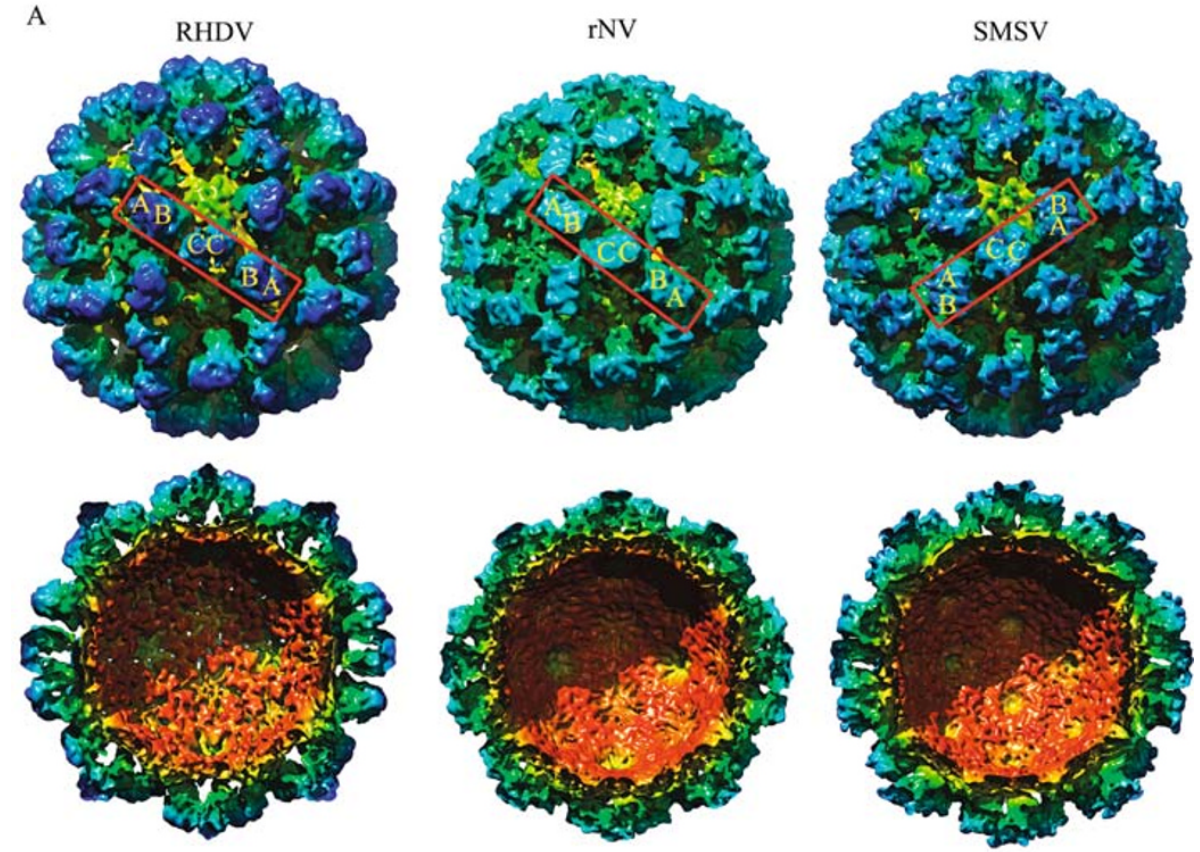

B

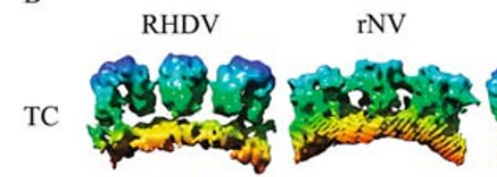

SMSV

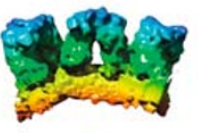

P2
P1
S

$\mathrm{CC}$
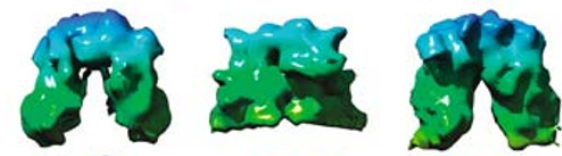

P2
P1

AB
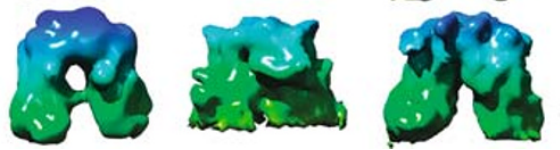

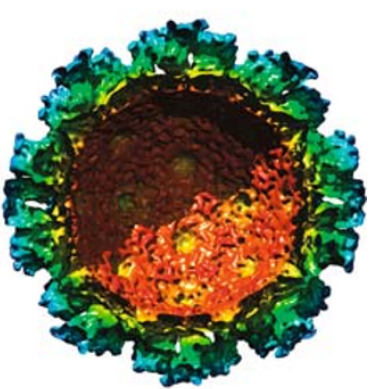

217
192
168
143
118

Figure 4. Structural comparisons among RHDV, rNV and SMSV. (A) The upper row shows the isosurface representations of RHDV, rNV and SMSV structures. The halves are shown in the bottom row. Three adjacent capsomers marked by red rectangles are separated out and further analyzed with side view in panel B. (B) Upper row (TC): side-views of three connected capsomers from the corresponding red box in panel A. Middle row (CC): side-views of CC capsomer. Bottom row (AB): side-views of $A B$ capsomer. Approximately, the density colored by blue/cyan represents $\mathrm{P} 2$ subdomain region, green represents $\mathrm{P} 1$ subdomain region and yellow/orange color for $S$ domain region. The color bar represents the different radii with four color gradients from red (118 $\AA$ ) to yellow (143 $\AA$ ), to green $(168 \AA)$, to cyan $(192 \AA)$ and to blue $(217 \AA)$.

compared with SMSV. Taken together, this comparison analysis reveals that the capsid proteins of caliciviruses have different conformations and could assemble into specific virions with different manners although they share certain sequence homology.

\section{CONCLUSIONS}

In summary, we have determined the cryo-EM structures of two types of wild RHDV particles, intact virion and the corelike particle (CLP), with the resolution $11 \AA$ and $17 \AA$, respectively. This is the first time to obtain the $3 \mathrm{D}$ structure of wild caliciviruses CLP, and the 3D structure of intact RHDV virion was the highest resolution structure in Lagovirus.

The structures of intact RHDV virion and the CLP show identical radical density distribution profile except the protrusion region. The absence of exact protrusion density in the CLP and the conserved jungle loop linking $P$ domain and $S$ domain of VP60 confirmed the fact that the CLP was just produced from the intact virion with the protrusion dissociated. This is the first time to confirm this hypothesis by structural approach. In addition, the jungle loop is highly 

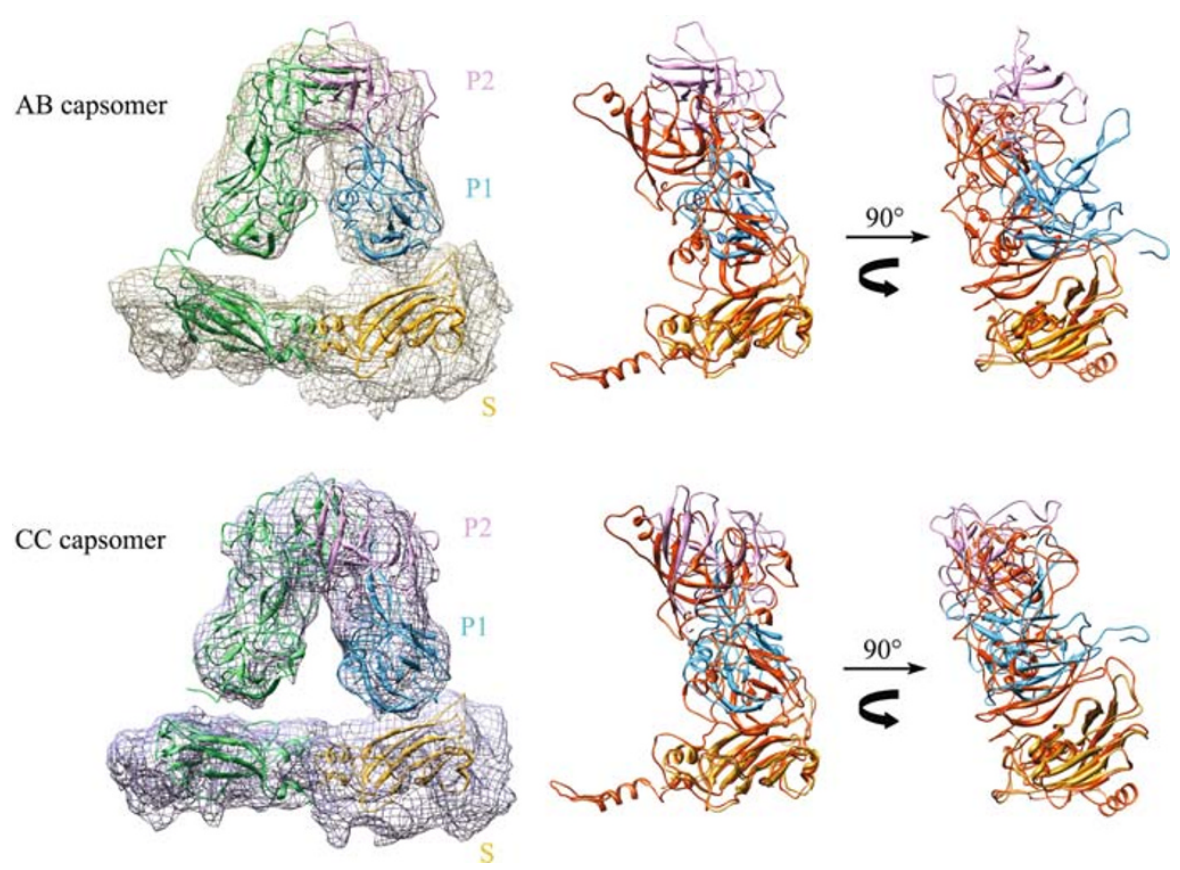

Figure 5. Modeling of RHDV AB and CC capsomers based on cryo-EM density and its comparison with SMSV major capsid protein VP60. The density of the AB/CC capsomer was segmented from the intact RHDV virion cryo-EM map, represented as mesh, and colored by grey/light blue. The structure of RHDV VP60 was modeled based on crystal structure of SMSV and fitted into AB or CC capsomer densities (see Materials and Methods). All structural models are represented as ribbon. The RHDV VP60 model is colored by green and SMSV VP60 by orange-red. The P1, P2 subdomains and S domain of RHDV VP60 are colored by dodger blue, plum and goldenrod, respectively. The VP60 S domains of RHDV and SMSV are superposed together to compare these two structures.

conserved among caliciviruses, suggesting the existence of the CLP in all caliciviruses species.

The specific structural features and assembly motifs of RHVD capsomers were revealed by comparison with the crystal structures of recombinant Norovirus and San Miguel sea lion virus. RHDV capsomer has a hat-shaped prominency at the $\mathrm{P} 2$ region, implying a specific antigen structure and antibody binding sites. In addition, the RHDV CC capsomer interacts with two adjacent $A B$ capsomers via $P 2$ subdomains, and no interactions were observed between $C C$ capsomer and two other adjacent $A B$ capsomers. Inside $A B$ capsomer, both $\mathrm{P} 1$ and $\mathrm{P} 2$ subdomains are involved in interactions, while only P2 subdomains are involved for CC capsomer. Comparison of the pseudo atomic models of RHDV VP60 based on EM map and the crystal structure of SMSV reveals the rotation of RHDV VP60 P domain with respect to its $S$ domain.

As a sub-family of the Caliciviridae family, there were limited structural researches on Lagovirus. Here, we took the advantage of the cryo-EM approach and revealed the structural features of Lagovirus. This study provides close insight into the structure of Lagovirus, which is important for future functional analysis and better vaccine development.

\section{MATERIALS AND METHODS}

\section{Purification of RHDV virion}

The RHDV strain "NJ85" was originally isolated in the Institute of Veterinary Science, Jiangsu Academy of Agricultural Sciences, China. Rabbits infected by RHDV died within $48 \mathrm{~h}$. The livers from infected rabbits were used for virus purification.

RHDV was purified as described before (Zheng et al., 2001) with a little modification. The rabbit liver was homogenized in PBS ( $\mathrm{pH} 7.2,1: 10, w / v)$ using a whirling blender and cell homogenizer. After low-speed centrifugation (8000 rpm, $40 \mathrm{~min}$ ), the supernatant containing virion was stirred at $4{ }^{\circ} \mathrm{C}$ overnight by adding solid polyethylenglycol 6000 to $6 \%(w / v)$ and solid $\mathrm{NaCl}$ to $3 \%(w / v)$. After low-speed centrifugation ( $8000 \mathrm{rpm}, 40 \mathrm{~min})$, the precipitate was resuspended in PBS and then combined with a mixture of butanol and isopentanol $(24: 1, \mathrm{v} / \mathrm{v})$ and stirred for $5 \mathrm{~min}$. The suspension was clarified by low-speed centrifugation (2500 rpm, $40 \mathrm{~min}$ ). The aqueous phase was collected and centrifuged at $15,000 \mathrm{~g}$ for $40 \mathrm{~min}$. The supernatant was then ultracentrifuged at $145,000 \mathrm{~g}$ for $2 \mathrm{~h}$ ( $45 \mathrm{Ti}$ rotor, Beckman). The pellets containing RHDV virions were collected, resuspended in TNE ( $0.05 \mathrm{~mol} / \mathrm{L}$ Tris, $0.05 \mathrm{~mol} / \mathrm{L} \mathrm{NaCl}$, and $0.005 \mathrm{~mol} / \mathrm{L}$ EDTA), overlaid on the cushion of $25 \%(w / v)$ sucrose solution in TNE and centrifuged at $145,000 \mathrm{~g}$ for $3 \mathrm{~h}$ (Beckman, $45 \mathrm{Ti}$ rotor). The 
precipitated virions were resuspended in TNE at $4^{\circ} \mathrm{C}$ for overnight. Concentration and quality of RHDV were verified by negative staining electron microscopy. The solution containing RHDV virion was fast frozen in liquid nitrogen and stored in liquid nitrogen before it was used for cryo-EM study.

\section{Electron microscopy, image processing and 3D reconstruction}

For negative stain electron microscopy, $5 \mu \mathrm{L}$ of purified RHDV sample was applied to a glow-discharged carbon-coated grid that was further stained with $5 \mu \mathrm{L}$ of $2 \%$ uranyl acetate. The stained sample was examined and imaged using an FEI Tecnai 20 electron microscope with electron source $\mathrm{LaB}_{6}$, operated at $200 \mathrm{kV}$. The images were recorded by Gatan UltraScan 894 CCD camera $(2 \mathrm{~K} \times 2 \mathrm{~K})$ with nominal magnification of 70,000 and the pixel size $2 \AA$.

For cryo-EM, RHDV sample was embedded in thin layer of vitreous ice on freshly carbon-coated holey EM grid by blotting the grids with filter paper and then plunging into liquid ethane cooled by liquid nitrogen. Frozen hydrated specimens were imaged by using FEI Tecnai 20 electron microscope $\left(\mathrm{LaB}_{6}\right)$ operated at $200 \mathrm{kV}$ with the low-dose mode (about $2000 \mathrm{e} / \mathrm{nm}^{2}$ ) and the nominal magnification of 50,000 . For each specimen area, the defocus was set to $1-2 \mu \mathrm{m}$. Images were recorded on Kodak SO163 films and then digitized by Nikon 9000 with the scanning step 2000 dpi corresponding to 2.54 angstrom/pixel. Particles were auto-selected using FindEM (Roseman, 2004). The defocus value of each micrograph was determined by CTFFIND3 (Mindell and Grigorieff, 2003) from the IMAGE 2000 suite (Crowther et al., 1996; Smith, 1999) and then the CTF of each micrograph was corrected using program "applyctf" from the EMAN suite (Ludtke et al., 1999). Image processing and 3D reconstruction were performed using EMAN (Ludtke et al., 1999) with Spider scripts (Frank et al., 1996; Shaikh et al., 2008) embedded for correspondence analysis (CORAN) of each image class, which was wrapped in the Appion package (Lander et al., 2009). The final reconstructed density map was further sharpened using an amplitude correction algorithm from the program EM-BFACTOR (Fernandez et al., 2008).

\section{Multiple sequence alignment}

The 23 sequences of the capsid protein or protein precursors of caliciviruses were obtained from NCBI (National Center for Biotechnology Information, http://www.ncbi.nlm.nih.gov) as described (Chen et al., 2004), including AY269825 (RHDV NJ85), M87661 (NV), U22498 (Mexico), AJ004864 (GrV), U07611 (Hawaii), X86557 (Lordsdale), AB042808 (Chiba), L23828 (KY89), U04469 (Desert Shield virus), AJ011099 (bovine), AF181082 (VESV), M87482 (SMSV4), AF091736 (pan-1), M87481 (SMSV1), M86379 (FCV-F9), U13992 (FCV-CFI), D31836 (FCV-F4), U65427 (Sapporo), X86560 (Manchester), U73124 (PV), AF182760 (porcine), Z69620 (EBHSV) and $\mathrm{AB} 00225$ (canine). Multiple-sequence alignment against these 23 sequences was calculated by using the program CLUSTALW2 (http://www.ebi.ac.uk/Tools/clustalw2/) (Larkin et al., 2007).

\section{Modeling and EM map fitting}

Based on sequences alignment above, quasi-atomic model of RHDV major capsid VP60 was built from SMSV crystal structure (Chen et al.,
2006) (pdb code: $2 \mathrm{GH} 8$ ) using the program Modeller (Eswar et al., 2006), and its $\mathrm{N}$-terminal arm ( $\mathrm{N}$-terminal 63 residues) was discarded. P1, P2 subdomain and S domain of RHDV VP60 were separated and fitted into $A B$ capsomer density, respectively. The fittings of $P 1$ subdomain and $S$ domain were achieved manually and further automatically optimized by UCSF Chimera (Pettersen et al., 2004). The fitting of P2 subdomain was made by hand, and its fitness was not accurate enough due to the variability of caliciviruses P2 subdomain and inaccuracy of P2 subdomain model of RHDV VP60. For CC capsomer fitting, the RHDV VP60 model was split into a P domain and $S$ domain which were fitted into the CC capsomer density, respectively, using the auto fitting program from UCSF Chimera (Pettersen et al., 2004). All the structure illustration, map segmentation and model fitting were created by the software package UCSF Chimera (Pettersen et al., 2004).

\section{DATABASE ENTRY}

The cryo-EM density maps of intact RHDV virion and CLP have been deposited in Electron Microscopy Data Bank (EMDB) with the access number EMD-5131 and EMD-5133, respectively.

\section{ACKNOWLEDGEMENTS}

We would like to thank Prof. Fang Wang from the Institute of Veterinary Science, Jiangsu Academy of Agricultural Sciences, for her kindness to supply us the liver tissue of infected rabbits. We would also like to thank Dr. Lingpeng Cheng (Institute of Biophysics, Chinese Academy of Sciences) for his help with image processing and operation of Chimera and Ruigang Su (F. Sun group) for his help on holey grids preparation. This work was supported by National Natural Science Foundation of China (Grant Nos. 30700029, 30721003), Chinese Academy of Sciences (KGCX1-YW-13), the National Basic Research Program (973 Program) (Nos. 2006CB806506, 2006CB911001) and the National Programs for High Technology Research and Development Program (863 Program) (No. 2006AA02Z173).

\section{ABBREVIATIONS}

ACSV, amyelois chronic stunt virus; CLP, core-like particle; CORAN, correspondence analysis; cryo-EM, cryo-electron microscopy; EBHSV, European brown hare syndrome virus; FCV, Feline calicivirus; FSC, Fourier shell correlation coefficient; GrV, Grimsby virus; HV, high density inside; LC, inner low density; LV, low density inside; MNV, Murine Norovirus; NV, Norovirus; ORF, open reading frame; PV, Parksville virus; RHD, rabbit hemorrhagic disease; RHDV, rabbit hemorrhagic disease virus; SMSV, San Miguel sea lion virus; VLPs, virus-like particles

\section{REFERENCES}

Alexandrov, M., Peshev, R., Bozhkov, S., Yanchev, I., and Doumanova, L. (1993). Electron- and immunoelectron-microscopic investigation on the rabbit haemorrhagic disease virus. Comp Immunol Microbiol Infect Dis 16, 21-27.

Antonio, L., and Lorenzo, C. (2008). How Many Caliciviruses are there in Rabbits? A Review on RHDV and Correlated Viruses. 
Lagomorph Biology 4, 263-278.

Barbieri I., Lavazza A., Brocchi E., Konig M., and Capucci, L. (1997). Morphological, structural and antigenic modifications of rabbit haemorrhagic disease virus in the course of the disease. In: Chasey D., Gaskell R.M., Clarke I.N. (eds) Proceedings of the 1st symposium on calicivirus of the European Society of Veterinary Virology (ESVV), Reading, UK, 182-193.

Barcena, J., Verdaguer, N., Roca, R., Morales, M., Angulo, I., Risco, C., Carrascosa, J.L., Torres, J.M., and Caston, J.R. (2004). The coat protein of Rabbit hemorrhagic disease virus contains a molecular switch at the $\mathrm{N}$-terminal region facing the inner surface of the capsid. Virology 322, 118-134.

Bertolotti-Ciarlet, A., White, L.J., Chen, R., Prasad, B.V., and Estes, M.K. (2002). Structural requirements for the assembly of Norwalk virus-like particles. J Virol 76, 4044-4055.

Bhella, D., Gatherer, D., Chaudhry, Y., Pink, R., and Goodfellow, I.G. (2008). Structural insights into calicivirus attachment and uncoating. J Virol 82, 8051-8058.

Chen, R., Neill, J.D., Estes, M.K., and Prasad, B.V. (2006). X-ray structure of a native calicivirus: structural insights into antigenic diversity and host specificity. Proc Natl Acad Sci U S A 103, 8048-8053.

Chen, R., Neill, J.D., Noel, J.S., Hutson, A.M., Glass, R.I., Estes, M. K., and Prasad, B.V. (2004). Inter- and intragenus structural variations in caliciviruses and their functional implications. J Virol 78, 6469-6479.

Clarke, I.N., and Lambden, P.R. (1997). The molecular biology of caliciviruses. J Gen Virol 78, 291-301.

Crowther, R.A., Henderson, R., and Smith, J.M. (1996). MRC image processing programs. J Struct Biol 116, 9-16.

Eswar, N., Webb, B., Marti-Renom, M.A., Madhusudhan, M.S., Eramian, D., Shen, M.Y., Pieper, U., and Sali, A. (2006). Comparative protein structure modeling using Modeller. Current protocols in bioinformatics/editorial board, Andreas, D.B. et al. Chapter 5, Unit 56.

Fernandez, J.J., Luque, D., Caston, J.R., and Carrascosa, J.L. (2008). Sharpening high resolution information in single particle electron cryomicroscopy. J Struct Biol 164, 170-175.

Frank, J., Radermacher, M., Penczek, P., Zhu, J., Li, Y., Ladjadj, M., and Leith, A. (1996). SPIDER and WEB: processing and visualization of images in $3 D$ electron microscopy and related fields. J Struct Biol 116, 190-199.

Granzow, H., Weiland, F., Strebelow, H.G., Liu, C.M., and Schirrmeier, H. (1996). Rabbit hemorrhagic disease virus (RHDV): ultrastructure and biochemical studies of typical and core-like particles present in liver homogenates. Virus Res 41, 163-172.

Hillman, B., Morris, T.J., Kellen, W.R., Hoffman, D., and Schlegel, D. E. (1982). An invertebrate calici-like virus. Evidence for partial virion disintegration in host excreta. J Gen Virol 60, 115-123.

Katpally, U., Wobus, C.E., Dryden, K., Virgin, H.W., 4th, and Smith, T. J. (2008). Structure of antibody-neutralized murine norovirus and unexpected differences from viruslike particles. J Virol 82, 2079-2088.

Lander, G.C., Stagg, S.M., Voss, N.R., Cheng, A., Fellmann, D., Pulokas, J., Yoshioka, C., Irving, C., Mulder, A., Lau, P.W., et al. (2009). Appion: an integrated, database-driven pipeline to facilitate EM image processing. J Struct Biol 166, 95-102.

Larkin, M.A., Blackshields, G., Brown, N.P., Chenna, R., McGettigan,
P.A., McWilliam, H., Valentin, F., Wallace, I.M., Wilm, A., Lopez, R., et al. (2007). Clustal $W$ and Clustal $X$ version 2.0. Bioinformatics 23, 2947-2948.

Laurent, S., Vautherot, J.F., Madelaine, M.F., Le Gall, G., and Rasschaert, D. (1994). Recombinant rabbit hemorrhagic disease virus capsid protein expressed in baculovirus self-assembles into viruslike particles and induces protection. J Virol 68, 6794-6798.

Liu, S.J., Xue, H.P., Pu, B.Q., and Qian, N.H. (1984). A new viral disease in rabbits. Anim Husb Vet Med 16, 253-255.

Ludtke, S.J., Baldwin, P.R., and Chiu, W. (1999). EMAN: semiautomated software for high-resolution single-particle reconstructions. J Struct Biol 128, 82-97.

Martin Alonso, J.M., Casais, R., Boga, J.A., and Parra, F. (1996). Processing of rabbit hemorrhagic disease virus polyprotein. J Virol 70, 1261-1265.

Meyers, G., Wirblich, C., and Thiel, H.J. (1991a). Genomic and subgenomic RNAs of rabbit hemorrhagic disease virus are both protein-linked and packaged into particles. Virology 184, 677-686.

Meyers, G., Wirblich, C., and Thiel, H.J. (1991b). Rabbit hemorrhagic disease virus-molecular cloning and nucleotide sequencing of a calicivirus genome. Virology 184, 664-676.

Mindell, J.A., and Grigorieff, N. (2003). Accurate determination of local defocus and specimen tilt in electron microscopy. J Struct Biol 142, 334-347.

Nowotny, N., Bascunana, C.R., Ballagi-Pordany, A., Gavier-Widen, D., Uhlen, M., and Belak, S. (1997). Phylogenetic analysis of rabbit haemorrhagic disease and European brown hare syndrome viruses by comparison of sequences from the capsid protein gene. Arch Virol 142, 657-673.

Ohlinger, V.F., and Thiel, H.J. (1991). Identification of the viral haemorrhagic disease virus of rabbits as a calicivirus. Rev Sci Tech 10, 311-323.

Pettersen, E.F., Goddard, T.D., Huang, C.C., Couch, G.S., Greenblatt, D.M., Meng, E.C., and Ferrin, T.E. (2004). UCSF Chimera-a visualization system for exploratory research and analysis. J Comput Chem 25, 1605-1612.

Prasad, B.V., Hardy, M.E., Dokland, T., Bella, J., Rossmann, M.G., and Estes, M.K. (1999). X-ray crystallographic structure of the Norwalk virus capsid. Science 286, 287-290.

Prasad, B.V., Matson, D.O., and Smith, A.W. (1994). Threedimensional structure of calicivirus. J Mol Biol 240, 256-264.

Roseman, A.M. (2004). FindEM-a fast, efficient program for automatic selection of particles from electron micrographs. J Struct Biol 145, 91-99.

Rossmann, M.G., and Johnson, J.E. (1989). Icosahedral RNA virus structure. Annu Rev Biochem 58, 533-573.

Shaikh, T.R., Gao, H., Baxter, W.T., Asturias, F.J., Boisset, N., Leith, A., and Frank, J. (2008). SPIDER image processing for singleparticle reconstruction of biological macromolecules from electron micrographs. Nat Protoc 3, 1941-1974.

Smith, J.M. (1999). Ximdisp-A visualization tool to aid structure determination from electron microscope images. J Struct Biol 125, 223-228.

Thouvenin, E., Laurent, S., Madelaine, M.F., Rasschaert, D., Vautherot, J.F., and Hewat, E.A. (1997). Bivalent binding of a neutralising antibody to a calicivirus involves the torsional flexibility of the antibody hinge. J Mol Biol 270, 238-246.

Valicek, L., Smid, B., Rodak, L., and Kudrna, J. (1990). Electron and 
immunoelectron microscopy of rabbit haemorrhagic disease virus (RHDV). Arch Virol 112, 271-275.

Venkataram Prasad, B.V., Hardy, M.E., and Estes, M.K. (2000). Structural studies of recombinant Norwalk capsids. J Infect Dis 181 Suppl 2, S317-321.

Wirblich, C., Thiel, H.J., and Meyers, G. (1996). Genetic map of the calicivirus rabbit hemorrhagic disease virus as deduced from in vitro translation studies. J Virol 70, 7974-7983.

Xu, Z.J., and Chen, W.X. (1989). Viral haemorrhagic disease in rabbits: a review. Vet Res Commun 13, 205-212.

Zheng, D., Xue, T., and Xu, W. (2001). Three-dimensional structure of the wild-type RHDV. Chinese Science Bulletin 46, 1005-1009. 